\title{
Fairness Evaluation in Cooperative Hybrid Cellular Systems
}

\author{
Juan F. Awad, Muhammad A. Imran and Rahim Tafazolli \\ Centre for Communication Systems Research \\ University of Surrey, Guildford, UK, GU2 7XH \\ Email: \{J.Awad, M.Imran, R.Tafazolli\}@surrey.ac.uk
}

\begin{abstract}
Many method has been applied previously to improve the fairness of a wireless communication system. In this paper, we propose using hybrid schemes, where more than one transmission scheme are used in one system, to achieve this objective. These schemes consist of cooperative transmission schemes, maximal ratio transmission and interference alignment, and non-cooperative schemes, orthogonal and non-orthogonal schemes used alongside and in combinations in the same system to improve the fairness. We provide different weight calculation methods to vary the output of the fairness problem. We show the solution of the radio resource allocation problem for the transmission schemes used. Finally, simulation results is provided to show fairness achieved, in terms of Jain's fairness index, by applying the hybrid schemes proposed and the different weight calculation methods at different inter-site distances.

Index Terms-Critical Users, Hybrid System, Maximal Ration Transmission, Interference Alignment, Radio Resource Management, Users Weights, Fairness.
\end{abstract}

\section{INTRODUCTION}

Wireless communication has developed dramatically over last decades giving rise to high data rate services such as multimedia applications, VoIP and high speed Internet on mobile devices. Unfortunately, the current communication system design has created a bottleneck for such high data rate communications for users at the cell edge or users who are experiencing deep shadowing and fading.

Orthogonal Frequency Division Multiplexing (OFDM), thanks to its various advantages, such as ability to fight InterSymbol Interference (ISI) and its robustness in frequency selective channels, is receiving more and more attention and has proven itself one of the most efficient multicarrier transmission techniques, such that the multiple access OFDM has been chosen as the air-interface for downlink in the next generation mobile communications. On the other hand, new challenges are introduced when employing OFDM, i.e. Radio Resource Management (RRM), which consists of distribution of subcarriers and power between users. RRM plays a key role in increasing the performance of wireless systems through utilising the available resource in the best way possible [1]-[3] in order to allocate subcarrier and power to users in accordance to the optimisation problem based on the computational ability of the hardware and the transmission scheme.

The fairness of cellular systems is taking increasing interests from systems designers. In general, difference channel conditions experienced means that some users will receive data rate much higher than others. Many methods already exists in the literature to achieve this fairness; Max-min fairness has been given in [2] which might achieve equal rate, but offer inflexible data rate distribution. Weighted sum rate methods, [4], while offering flexible fairness, by adjusting weights, it gives no guarantee for meeting proportional fairness. Last but not least, proportional fairness, [5], maintain proportional rates among users according to a preset values.

In this paper, we will propose grouping users in two distinct groups as critical and non critical to improve fairness, each group is served using a different transmission scheme. This can be categorised as a frequency reuse method that differs from other frequency reuse methods already in the literature, like Fractional Frequency Reuse (FFR) [6] and Soft Frequency Reuse (SFR) [7]. Critical users are the vulnerable users located far from the base station and they usually require schemes that avoid or orthogonalise the interference. This is done in this paper by serving the critical users using maximal ratio transmission and interference alignment where they use a subband orthogonal to that used by the non-critical users at the cell centre. This in turn will eliminate the interference between the two schemes. Maximal Ration Transmission (MRT), is a transmit diversity technique [8] capable of maximizing the received signal power at the receiver. Whereas, interference alignment is a linear precoding technique to align all the interference on one or more frequency, time or space dimension. although it has been recently found as a coding technique by [9], but then it was employed as a transmission scheme by the authors in [10] and [11].

We will organise the rest of this paper as follows: In Section III we define our system model and formulate the problem. In Section III, different weight calculation methods used in this paper are defined. Additionally, power and subcarrier allocation methods to solve the formulated problem are given in Section IV In Section D, simulation results showing the fairness achieved while using multiple transmission schemes and multiple weight calculation methods. Finally, in Section VI] we conclude this paper.

\section{System Model And Problem Formulation}

\section{A. System Model}

We assume to have downlink system scenario consists of many clusters and each cluster is made up from three base stations sectors. Each sector is served by an antenna located 
on the outer corners of the hexagonally-shaped sectors. The system model is depicted in fig. 1 All base stations are connected to a Central Processing Unit (CPU) through a fibre optic backhaul which is assumed to have an unlimited bandwidth. The CPU has the function of radio resource management and scheduling decisions which requires collaboration between base stations. The channel is considered to be frequency-selective channel with slow fading. We assume that channel state information (CSI) is available globally and instantaneously at all the decision-making terminals. CSI is estimated at the receiver and sent back to the transmitter in a timely manner allowing accurate and current CSI.

In this paper, we consider hybrid system where two transmission schemes are used to serve the users; one for the noncritical user and other for the critical users. Critical users are the users located further away from their respective base station towards the centre of the cluster and they are called critical due to the bad channel condition they experience caused by shadowing and path loss. This is shown in Figure 11 In the hybrid scheme, non-critical users are served using non-cooperative schemes; orthogonal (frequency reuse factor of 3) or non-orthogonal (frequency reuse factor of 1) scheme whereas critical users are served using cooperative maximal ratio transmission, and interference alignment or orthogonal schemes. Each user has a a corresponding weight which is used as a method to achieve different level of fairness as explained in Section III

The interference caused by the adjacent clusters is ignored and considered to be part of the Additive Gaussian White Noise (AWGN). The available spectrum at the cluster is split into two orthogonal sub-bands, one for the non-critical users' region $f^{n}$ and the other for the critical users' region $f^{c}$. Consecutively, sub-bands are in turn divided into three smaller sub-bands $f_{1}^{n}, f_{2}^{n}$ and $f_{3}^{n}$ between the three sectors if orthogonal scheme is employed or utilised undivided if any of the other remaining schemes is used. An illustrative example of the frequency reuse is shown in figure 2 Likewise, available power is divided between the two regions. Part of the total power $P_{m}^{T}$ is given to users in the non-critical regions, $P_{m}^{n}$, and the other part is given to users in the critical region $P_{m}^{c}$ of sector $m$ where $P_{m}^{T}=P_{m}^{n}+P_{m}^{c}$.

\section{B. Problem Formulation}

In this paper, the objective is to maximise the weighted sum rate of the cluster, one sector at a time, according to power constraints. The mathematical representation of the total weighted sum rate, for the whole system, optimisation problem of base station $m$ can be written as:

$$
\begin{aligned}
\max _{\mathbf{P}} & \sum_{k \in \mathcal{K}} w_{k} R_{k} \\
\text { subject to : } & p_{k, n} \geq 0 \quad \forall n, k \in \mathcal{N}, \mathcal{K} \\
& \sum_{\mathcal{N}} \sum_{\mathcal{K}} p_{k, n} \leq P_{m}, \forall m,
\end{aligned}
$$

where $w_{k}$ is the weights corresponds to user $k, P_{m}$ is the total power available at base station $m . \mathcal{N}$ and $\mathcal{K}$ are the sets of

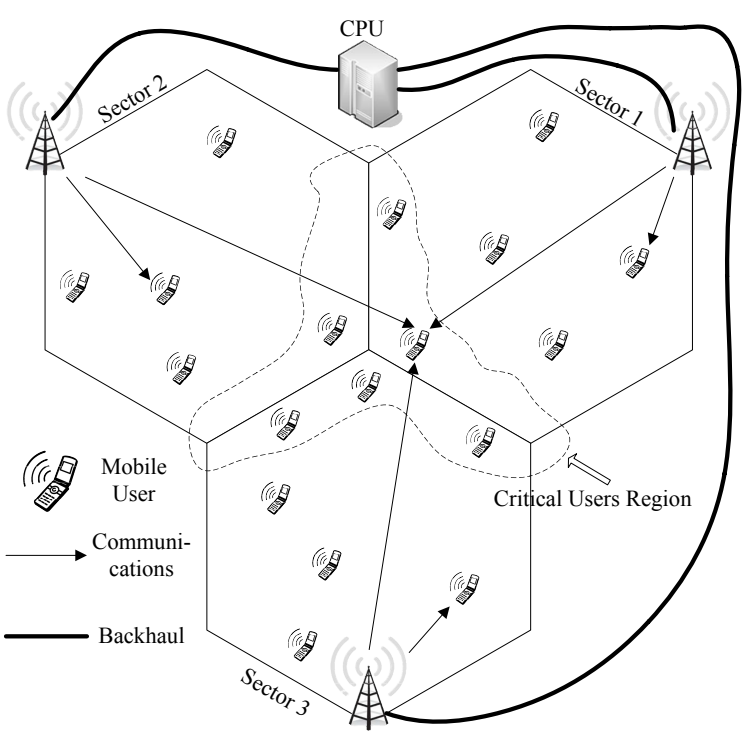

Fig. 1. System Model

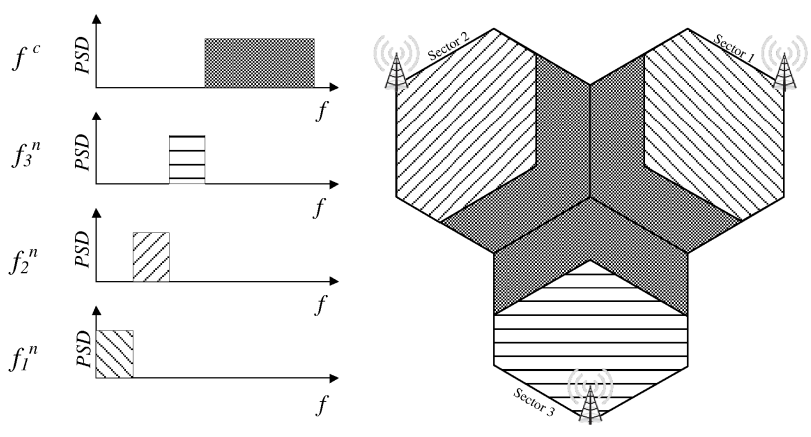

Fig. 2. Bandwidth Division

users and subcarriers, respectively, available at their respective sector. $p_{k, n}$ is the transmit power to user $k$ on subcarrier $n$. In this paper, the weights $w_{k}, \forall k=1, \ldots, K$ are assigned according to a predetermined methods discussed in Section III.

In the case of hybrid transmission schemes the problem of the total weighted sum rate becomes the sum of two independent problems each represent one of the two transmission schemes. While each transmission scheme had a different rate calculation method, they are not coupled together so the problem in (1) is decomposable as follows: 


$$
\begin{aligned}
\max _{\mathbf{P}} & \sum_{k \in \mathcal{K}} w_{k} R_{k}=\sum_{k \in \mathcal{K}^{n}} w_{k} R_{k}^{n}+\sum_{k \in \mathcal{K}^{c}} w_{k} R_{k}^{c} \\
\text { subject to : } \quad & p_{k, n} \geq 0 \quad \forall n, k \in \mathcal{N}^{n}, \mathcal{K}^{n} \\
& p_{k, n} \geq 0 \quad \forall n, k \in \mathcal{N}^{c}, \mathcal{K}^{c} \\
& \sum_{\mathcal{N}^{n}} \sum_{\mathcal{K}^{n}} p_{k, n} \leq P_{m}^{n}, \forall m \\
& \sum_{\mathcal{N}^{c}} \sum_{\mathcal{K}^{c}} p_{k, n} \leq P_{m}^{c}, \forall m,
\end{aligned}
$$

where $\sum_{k \in \mathcal{K}^{n}} w_{k} R_{k}^{n}$ is the total weighted sum rate for noncritical users whereas $\sum_{k \in \mathcal{K}^{n}} w_{k} R_{k}^{c}$ is the total weighted sum rate for critical users. The formulae of $R_{k}^{n}$ and $R_{k}^{c}$ are dependent on the transmission scheme used for that specific set of users. $\mathcal{N}^{n}$ and $\mathcal{N}^{c}$ are the sets of subcarriers that are assigned to the non-critical and critical users, respectively. Likewise, $\mathcal{K}^{n}$ and $\mathcal{K}^{c}$ are the sets non-critical and critical users, respectively. $P_{m}^{n}$ and $P_{m}^{c}$ are the total power assigned to the non-critical and critical users in sector $m$, respectively. For the rest of this paper, a superscript of $n$ indicates a variable or a vector that corresponds to the non-critical users and a superscript of $c$ correspond to one related to the critical users.

\section{Weight Calculation Methods}

In this paper, we will consider three different methods to calculate the weights corresponding to each user. Each method will correspond to a different objective. The effect of each method will be shown in Section $\mathrm{V}$. These weights are not a result of an optimisation problem, in the contrary they are constant if all the parameter associated with the method of calculation are constant. These methods are:

1) Ones: weights are all equal to one giving no priority to any users and it is a good as a benchmark for comparison.

2) Inverse of Pathloss and Shadowing: Users weights are inversely proportional to the Distance-Dependent Pathloss (DDPL) and shadowing $w_{k}=(S P)^{-1}$ where $S$ is the shadowing value and $P$ is the pathloss value. Using this technique, large weights are given to users far away from the base station or are located in deep shadowing region.

3) Previous Average Rates: Here, we calculate the average rates from a windows of certain number of previous channel realisations $L . w_{K}=\left(\frac{1}{l} \sum_{l=1}^{L} R_{k}^{l}\right)^{-1}$. The better the previous throughput of some users the lower their weight will be and vice versa, allowing users with poor throughput to get more resources.

All weights are normalised so $\sum_{\mathcal{K}} w_{k}=|\mathcal{K}|$.

\section{Radio Resource Allocation}

\section{A. Orthogonal and Non-Orthogonal}

Those both schemes are similar, the only difference lies in the frequency reuse. Orthogonal scheme has a frequency reuse factor of 3 where the available frequency band is divided into three smaller orthogonal sub-bands between the three sectors. Whereas, the non-orthogonal scheme has a frequency reuse factor of 1 where all of the available band is used in all sectors. That in turn lead inter sector interference. The rate of user $k$ in sector $m$ for the orthogonal and non-orthogonal schemes can formulated as follows:

$$
R_{k}=\sum_{n \in \mathcal{N}_{k}} B_{N} \log _{2}\left(1+\frac{p_{k, n, m}\left|h_{k, n, m}\right|^{2}}{N_{0} B_{N}+\sum_{l \neq m} p_{k, n, l}\left|h_{k, n, l}\right|^{2}}\right),
$$

where $\mathcal{N}_{k}$ is the set of subcarriers assigned to user $k, h_{k, n, m}$ is the complex channel from base station $m$ to user $k$ in sector $m$ on subcarrier $n$ and $p_{k, n, m}$ is the power transmitted along this channel from base station $m$ to user $k$ on subcarrier $n$. $B_{N}$ and $N_{0}$ are the bandwidth per subcarrier and the noise spectral density, respectively. The term $\sum_{l \neq m} p_{k, n, l}\left|h_{k, n, l}\right|^{2}$ represent the interference caused by transmitting signal from other base stations on the same subcarrier. This term is equal to zero in orthogonal scheme because subcarriers are unique in each base station therefore orthogonal.

For the subcarrier allocation for both of these schemes, we use the algorithm given in [2] where each user with the lowest data rate is assigned a subcarrier iteratively until subcarriers are assigned. As for the power control, we use the well known single user water-filling [12] and the power assigned to user $k$ on subcarrier $k$ in sector $m$ is

$$
p_{k, n, m}=\left[\frac{w_{k}}{\lambda}-\frac{1}{H_{k, n, m}}\right]^{+} \quad \forall k, n, m,
$$

$H_{k, n, m}=\frac{\left|h_{k, n, m}\right|^{2}}{N_{0} B_{N}}$ is the unit power channel gain, and we define $[x]^{+}=\max (0, x)$.

\section{B. Maximal Ratio Transmission}

In this scheme, the three base stations transmit on the same subcarrier to one user and the received signals is then added constructively. The benefit of MRT is that it increase diversity gain, and make benefit from the interference caused by other base stations. The disadvantage of this scheme lies in the complexity where global channel knowledge is needed to achieve the diversity gain, in addition high level of synchronisation is also required. While using MRT, the whole cluster is considered to be one sector and the three base station will constitute Distributed-MIMO, [13], with the help of the CPU. All users will be become part of one system and do not belong to certain base station. The data rate of user $k$ is calculated as follows, [8]:

$$
R_{k}=\sum_{n \in \mathcal{N}_{k}} B_{N} \log _{2}\left(1+\sum_{m \in \mathcal{M}} p_{k, n, m} \frac{\left|h_{k, n, m}\right|^{2}}{N_{0} B_{N}}\right),
$$

where $\mathcal{M}$ is the set of base stations.

To assign the subcarrier, we use the same method in [2], but in a different context. Because each user has to be connected to the three base stations through the same subcarrier, we 
apply the algorithm to the average SNR experienced on the three links. Where $\operatorname{avg}\left(\mathrm{SNR}_{k, n}\right)=\frac{1}{3} \sum_{m=1}^{3} \mathrm{SNR}_{k, n, m}$ and the choice of subcarriers is related to $\operatorname{avg}\left(\mathrm{SNR}_{k, n}\right)$. Power allocation problem is found to be a water-filling problem, [14]:

$$
p_{k, n, m}=\left[\frac{w_{k}}{\gamma}-\frac{1}{H_{k, n, m}}\right]^{+} \quad \forall k, n, m,
$$

where $\gamma=\sum_{m=1}^{M} \lambda_{m}$ is the water level. Equation (6) is a single user water-filling at each base station making the power allocation problem distributed and reducing the computational complexity at the CPU.

\section{Interference Alignment}

In frequency domain, the interference alignment is achieved by considering subcarriers as separate dimensions, called symbol extensions. It is found in [10] that using interference alignment in an interference channel, each user is able to achieve half of the degrees of freedom achieved in the interference free channel. To be able to achieve this degree of freedom, we construct, from our three-sector scenario, multiple 3-user interference channels each consists from the three base stations and three users. Resource allocation procedure for the interference alignment is slightly different where after assigning the subcarrier, we need to find the precoding and interference suppression vectors before performing the power allocation.

For each of the 3 -user interference channels, a $3 / 2$ degrees of freedom can be achieved and requires and this requires two transmit and receive dimensions. We divide the available subcarriers $\mathcal{N}$, assuming $|\mathcal{N}|$ is even, into two equal, smaller, subsets of subcarriers $\mathcal{N}_{1}$ and $\mathcal{N}_{2}$ where $\left|\mathcal{N}_{1}\right|=\left|\mathcal{N}_{2}\right|$ and $\mathcal{N}_{1} \cap \mathcal{N}_{2}=\phi$. Users are assigned pairs of subcarriers, each is chosen from one of the subsets $\mathcal{N}_{1}$ and $\mathcal{N}_{2}$. Subcarriers are chosen according the to the algorithm in [2] from each subset at a time. And the equivalent channel from base station $j$ to user $k$ in the three user interference channel on the subcarrier pair $\left\{n_{1}, n_{2}\right\}$

$$
H_{m k}^{n_{1} n_{2}}=\left[\begin{array}{cc}
h_{m k}^{n_{1}} & 0 \\
0 & h_{m k}^{n_{2}}
\end{array}\right]
$$

where $h_{m k}^{n_{1}}$ is the complex channel from base station $m$ to user $k$ on subcarriers $n_{1}$ and $h_{m k}^{n_{1}}$ on subcarrier $n_{2} . n_{1} \in \mathcal{N}_{1}$ and $n_{2} \in \mathcal{N}_{2}$. For simplicity, we define $\mathcal{N}^{p}=\left\{n_{1}^{p}, n_{2}^{p}, \ldots, n_{n_{c}}^{p}\right\}$ as the set of subcarrier pairs.

The next step is to calculate the precoding and interference suppression vectors, this can be done using different methods that already exist in the literature. An analytical method to calculate the precoding vectorsonly, only, was given in [10]. The authors in [15] proposed two iterative algorithm to solve this problem. A distributed leakage minimisation (leak-min) algorithm that depends on the channel reciprocity and na SINR maximising (max-SINR) algorithm. A rank constrained rank minimisation (RCRM) method was proposed by [16] which is, unfortunately, computationally extensive. A comparison between the three algorithms for symbol extended transmissions was shown in [16] where RCRM performed best and leak-min worst. In this paper, we will use the leak-min algorithm, despite its relatively poor performance, because of it's distributed property and relative robustness. The transmitted signal is pre-multiplied by the $2 \times 1$ precoding vector $V_{j}$ at transmitter $j$ and then, at receiver $k$, the received signal is multiplied by the $1 \times 2$ interference suppression vector $U_{k}$ and the $1 \times 1$ equivalent channel from $j$ to $k$ becomes:

$$
h_{j k}^{n_{p}}=U_{k} H_{j k} V_{j} .
$$

The rate calculation equation for the interference alignment scheme on subcarrier pair $n^{p}$

$$
R_{k}=\sum_{n^{p} \in \mathcal{N}_{k}^{p}} B_{N} \log _{2}\left(1+\frac{p_{k, n^{p}}\left|h_{k k}^{n^{p}}\right|^{2}}{N_{0} B_{N}+\sum_{j \neq k} p_{j, n^{p}}\left|h_{j k}^{n^{p}}\right|^{2}}\right)
$$

To solve for the power allocation problem, we substitute (9) in (1). We assume that the leak-min algorithm is able to completely remove the residual interference, the interference in (9) goes to zero and the solution for the power allocation problem is:

$$
p_{k, n^{p}}=\left[\frac{1}{\lambda}-\frac{1}{\tilde{H}_{k, n^{p}}}\right]
$$

where $\lambda$ is the water level and $\tilde{H}_{k, n^{p}}=\frac{\left|h_{k k}^{n^{p}}\right|^{2}}{N_{0} B_{N}}$. Equation (10) is a water-filling equation at each base station and this a distributed solution for interference alignment problem. The solution in this paper is in line with the results found in [17].

\section{Simulation Results}

All results presented in this paper corresponds to a frequency-selective, slow fading channel model in line with the ITU Pedestrian B model [18]. The simulation parameters are shown in Table I. Users in these simulations are distributed in a equally spaced pre-determined positions. Fairness in this section is measured using Jain's fairness [19] which can be defined as:

$$
\mathcal{J}\left(r_{1}, r_{2}, \ldots, r_{K}\right)=\frac{\left(\sum_{k=1}^{K} r_{k}\right)^{2}}{K \cdot \sum_{k=1}^{K} r_{k}^{2}}
$$

Jain's fairness index ranges from $\frac{1}{K}$, which is the worst case, to one, the best case. The maximum index achieved when all the users have equal rates. While Jain's fairness index in not the only fairness index, is one of the most commonly used. Other fairness measures include [20] and the empirical Cumulative Distribution Function (CDF) plot. Showing the CDF plots for each case is not possible due to size constraints. For compactness, we donate $w^{(1)}$ for the weight calculating method when all the weights are one, $w^{(2)}$ when the weights are an inverse of the distance dependent path loss and shadowing, and $w^{(3)}$ for the rate related weight calculation method. 

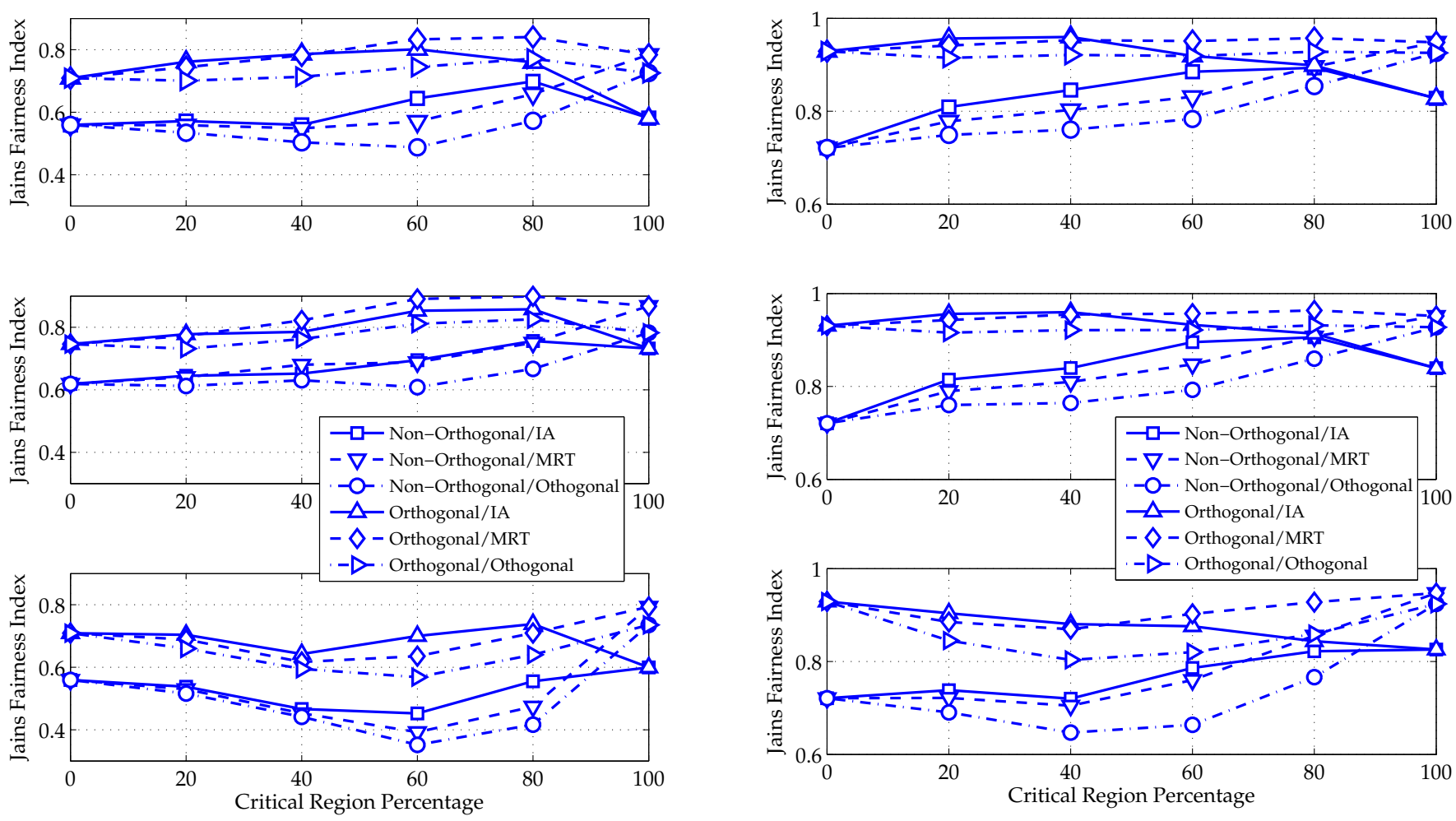

Fig. 3. Fairness Analysis at ISD 3000m

Fig. 4. Fairness Analysis at ISD 1000m

TABLE I

SYSTEM PARAMETERS

\begin{tabular}{|c|c|}
\hline Parameter & Value \\
\hline \hline Number of subcarriers & 128 \\
\hline Number of users in each sector & 12 \\
\hline Max power at Each Base Station & $50 \mathrm{~W}$ \\
\hline Bandwidth & $20 \mathrm{MHz}$ \\
\hline Noise PSD & $-139 \mathrm{dBm}$ \\
\hline Path Loss Exponent & 3.5 \\
\hline Shadowing Standard Deviation & $8 \mathrm{~dB}$ \\
\hline Multipath Model & ITU Ped. B \\
\hline Inter-Site Distance & 1,3 or $5 \mathrm{~km}$ \\
\hline Users' distribution & Deterministically \\
\hline
\end{tabular}

To show the main essence of this paper, we divide system into two regions each served with a different transmission scheme. A region for the critical users and another for the noncritical users. We define the non-critical users region by the region where users located less than a certain percentage from the BS to the Clusters Mid-Point (CMD). For example, assuming $40 \%$ of the sectors is for the critical users, that indicates that users located less $40 \% \times$ (Distance from BS to CMD) are considered to be non-critical. Additionally, resources (i.e. power and subcarriers) are divided between the two regions proportionally to the percentage of users in each corresponding scheme.

In this part of the paper, we show the fairness of each combination of schemes mentioned previously and at different weight calculation methods. Figures 4, 3 and 5] show Jain's fairness index when the inter-site distance is is $1000 \mathrm{~m}, 3000 \mathrm{~m}$ and $5000 \mathrm{~m}$, respectively, and are plotted against the percentage of users in the critical scheme. In each figure, the top subfigure corresponds to the weight calculation method $w^{(1)}$, the middle one corresponds to $w^{(2)}$ and, the bottom one corresponds to $w^{(3)}$.

In general, the fairness achieved when the non-critical users are served served using the orthogonal scheme is much higher that the non-orthogonal scheme. This is expected due to the absence of interference. The hybrid scheme that achieve the best fairness result in most scenarios is the Orthogonal/MRT scheme. In the MRT case, the interference is eliminated and transmit diversity gain is achieved which help provide fairer rate to the users regardless of their locations in the sector. It is observed from Figs. 45 that different percentages of critical users can achieve the highest level of fairness, but considering $80 \%$ of the users critical achieves best fairness in most scenarios. This is partly due to the greedy resource allocation approach when the group of non-critical users is large, resources will be given to small number of users with high channel gains near the BS. Moreover, when all the users are critical, the cooperative scheme will behave similar to the non-cooperative scheme due to the same greedy resource allocation. 


\section{REFERENCES}
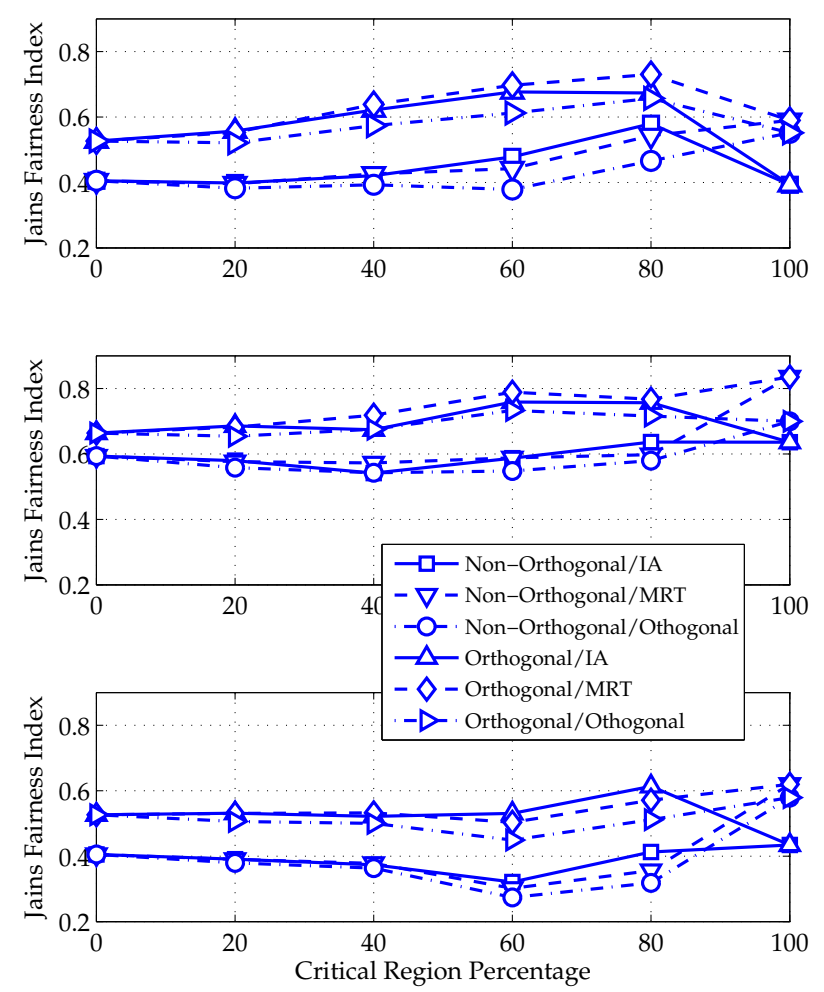

Fig. 5. Fairness Analysis at ISD 5000m

It is worth noting that when using rate-related weight method, $w^{(3)}$, the fairness drops considerably due to the aggressive nature of this method and the sensitivity of Jain's fairness index. Rate-related weights method, gives very high weights to the users with bad performance and almost zero rates to the users with high performance. This near-zero weights renders the resource allocation to those users unfeasible. The weight calculation method $w^{(2)}$ achieves the best fairness measure over all the transmission schemes and intersite distances.

\section{CONCLUSION}

In this paper, the fairness of a three sector scenario is evaluated. We proposed hybrid transmission schemes where more than one transmission scheme are used. Each sector is divided into two regions, critical and non-critical users region. Additionally, resource allocation approaches are clarified for the transmission schemes used, particularly the cooperative one, maximal ratio transmission and interference alignment. Furthermore, three methods of weight calculation are used to compare their effect on the system overall fairness. Finally, simulation results for the hybrid transmission scheme proposed alongside different weight calculation methods are used at multiple inter-site distances. Results showed the superiority of the orthogonal scheme when serving the non-critical users. And with MRT for the critical users they achieve the best fairness measure in most scenarios.
[1] J. Jang and K. Bok Lee, "Transmit power adaptation for multiuser OFDM systems," IEEE Journal on Slected Areas in Communications, vol. 21, no. 2, pp. 171-178, February 2003.

[2] W. Rhee and J. Cioffi, "Increase in capacity of multiuser OFDM system using dynamic subchannel allocation," in IEEE 51st Vehicular Technology Conference Proceedings, vol. 2, 2000, pp. 1085-1089.

[3] S. Sadr, A. Anpalagan, and K. Raahemifar, "Radio resource allocation algorithms for the downlink of multiuser ofdm communication systems," IEEE Communications Surveys Tutorials, vol. 11, no. 3, pp. 92-106, Third Quarter 2009.

[4] R. Cendrillon, M. Moonen, J. Verlinden, T. Bostoen, and W. Yu, "Optimal multiuser spectrum management for digital subscriber lines," in IEEE International Conference on Communications Proceedings, vol. 1, July 2004, pp. 1-5.

[5] Z. Shen, J. G. Andrews, and B. L. Evans, "Adaptive resource allocation in multiuser OFDM systems with proportional rate constraints," IEEE Transactions on Wireless Communications, vol. 4, no. 6, pp. 2726-2737, November 2005.

[6] M. Sternad, T. Ottosson, A. Ahlen, and A. Svensson, "Attaining both coverage and spectral efficiency with adaptive OFDM downlinks," in Proceedings of The 58th IEEE Semiannua Vehicular Technology Conference, 2003. VTC 2003-Fall., October 2003.

[7] Huawei, Soft Frequency Reuse Scheme. UTRAN LTE, 3GPP R1050507, TSG RAN WG1 Meeting 41, Athens, Greece, May 2007.

[8] A. Goldsmith, Wireless Communications. Cambridge University Press, 2005.

[9] M. Maddah-Ali, A. Motahari, and A. Khandani, "Signaling over mimo multi-base systems: Combination of multi-access and broadcast schemes," in Information Theory, 2006 IEEE International Symposium on, Jul. 2006, pp. 2104-2108.

[10] V. Cadambe and S. Jafar, "Interference alignment and degrees of freedom of the $K$-user interference channel," Information Theory, IEEE Transactions on, vol. 54, no. 8, pp. 3425-3441, Aug. 2008

[11] — , "Interference alignment and the degrees of freedom of wireless $X$-networks," Information Theory, IEEE Transactions on, vol. 55, no. 9, pp. 3893-3908, Sept. 2009.

[12] J. Jang and K. B. Lee, "Transmit power adaptation for multiuser OFDM systems," IEEE Journal on Selected Areas in Communications, vol. 21 no. 2, pp. 171-178, February 2003.

[13] J. Wang, F. Adachi, and X. Xia, "Coordinated and distributed mimo [guest editorial]," IEEE Wireless Communications, vol. 17, no. 3, pp. 24-25, June 2010.

[14] J. Awad, M. Imran, and R. Tafazolli, "Improving fairness by cooperative communications and selection of critical users," in Wireless Communications and Mobile Computing Conference (IWCMC), 2011 7th International, July 2011, pp. 184-188.

[15] K. Gomadam, V. Cadambe, and S. Jafar, "A distributed numerical approach to interference alignment and applications to wireless interference networks," Information Theory, IEEE Transactions on, vol. 57, no. 6, pp. 3309-3322, Jun. 2011.

[16] D. S. Papailiopoulos and A. G. Dimakis, "Interference alignment as a rank constrained rank minimization," arXiv.org, vol. abs/1010.0476, 2010, http://arxiv.org/abs/1010.0476

[17] B. Da and R. Zhang, "Exploiting interference alignment in multicell cooperative ofdma resource allocation," in 2011 IEEE Global Telecommunications Conference (GLOBECOM 2011), Dec. 2011, pp. $1-5$.

[18] "Recommendation ITU-R M.1225: Guidelines for evaluation of radio transmission technologies for IMT-2000," Tech. Rep., 1997.

[19] R. Jain, D. Chiu, and W. Hawe, "A quantitative measure of fairness and discrimination for resource allocation in shared computer systems," DEC Research, Tech. Rep. TR-301, September 1984.

[20] C. W. Gini, "Variabilitè e mutabilitè," Studi Econornico-Giuridici della R. Universita de Cagliari, 1912. 